\title{
PREFERENCE MATRICES IN TROPICAL ALGEBRA
}

\author{
Hana Tomášková \\ University of Hradec Králové, Faculty of Informatics and Management, \\ Rokitanského 62, 50003 Hradec Králové, \\ Czech Republic \\ e-mail: hana.tomaskova@uhk.cz \\ Martin Gavalec \\ University of Hradec Králové, Faculty of Informatics and Management, \\ Rokitanského 62, 50003 Hradec Králové, \\ Czech Republic \\ e-mail: martin.gavalec@uhk.cz
}

\begin{abstract}
Relative importance of alternatives in AHP multi-criteria decision problem is standardly computed from the (possibly incosistent) preference matrix as the eigenvector of the preference matrix by methods of linear algebra. Alternative use of non-standard methods in other algebras, such as tropical or fuzzy algebra is considered in this paper. The preference matrix is investigated by the methods used in max-prod algebra. Given preference matrix is processed by max-prod operations, until a steady state is reached. The eigenvector of the matrix then describes the steady state preferences and respects all preference relations contained in the original matrix. Efficient algorithms for computing eigenvectors in the tropical algebra are described. The method is illustrated by numerical examples and compared with the linear algebra approach. The consistent and inconsistent cases are considered.
\end{abstract}

Keywords: preference matrix, tropical algebra, AHP decision making, eigenvectors.

JEL classification: C44

AMS classification: $90 \mathrm{C} 15$

\section{Introduction}

Analytical Hierarchy Process is a mathematical model of multi-criteria decision making that uses the decomposition of a complex unstructured situation into simpler parts - the hierarchical system. Using subjective pairwise comparisons numerical values are assigned to individual components, showing their relative importance. One of the fundamental questions in AHP decision making is how to find the appropriate preference matrix for a set of alternatives.

The subjective preferences given by human experts are often inconsistent and do not reflect the deep relations between the processed notions, see [Ramík \& Korviny, 2010, Ramík \& Perzina, 2010]. The standard approach to finding the relative importance vector out of an incosistent matrix uses an eigenvector of the preference matrix computed by the methods of linear algebra, [Saaty, 2003]. Possible use of other non-standard methods in other algebras, such as tropical or fuzzy algebra, is considered in this paper. Differently from binary operations plus and product in classical linear algebra, the tropical algebra uses binary operation maximum and plus, or maximum and product, or similar combinations with minimum instead of maximum. Similarly, the fuzzy algebra uses the operation maximum together with some fuzzy triangular norm, e.g. the Gödel, Łukasiewicz, product, or drastic t-norm, [Carlsson \& Fullér, 1996, Leung \& Cao, 2000]. Properties of various tropical and fuzzy algebras were studied by many authors, with useful applications. Tropical algebras are important in the study of discrete events systems, the steady states of which correspond to eigenvectors of max-plus or max-prod matrices, see [Brualdi \& Ryser, 1991, Butkovič, 2010, Cuninghame-Green, 1976, Heidergott et al., 2006, Olsder, 1991]. The eigenvectors of max-min matrices are useful in cluster analysis or in fuzzy reasoning, the eigenvectors of some fuzzy 
algebras are described in [Rashid et al., 2012, Gavalec et al., submitted].

In this paper the preference matrices is studied by methods used in tropical algebra, in particular in the max-prod algebra. The original preference matrix is processed by max-prod operations, until a steady state is reached. The eigenvector of the matrix then describes the steady state preferences and, therefore, it respects all relations hidden in the original matrix. Efficient algorithms for computing eigenvectors in the tropical algebra are used. The method is illustrated by numerical examples and compared with the linear algebra approach. The consistent and inconsistent cases are considered separately.

\section{AHP decision making}

Analytic Hierarchy Process (AHP) is a method developed for creating structured models of multi-criteria decision problems. The method helps to find an alternative which suits best the given needs of the deciding person. Analyzing the set of possible alternatives, the AHP method finds the one with the best rating, based on the structure of the problem and given preferences. Saaty formulated the principles of AHP in late 1970s [Saaty, 1980], and the method has been broadly studied and applied in many cases since the time, [Dytczak \& Szklennik, 2011, Mls \& Gavalec, 2009, Ramík \& Perzina, 2010]. The method combines mathematical and psychological aspects, starting with defining the structure of the problem, then quantifying the relative preferences, computing the priorities and finally computing the evaluation of all considered alternatives. First of all, the multi-criteria decision problem is converted into a hierarchy of sub-problems and each of the sub-problems is then independently analyzed. The criteria of the subproblems in the hierarchy may have very heterogeneous nature, they may be precisely or vaguely defined, with crisp or fuzzy parameters, formal or intuitive, etc. The relative preferences of heterogeneous criteria are then quantified by human decision-maker using the ability to compare various aspects of the problem. The decision maker systematically compares the criteria in pairs and quantifies the relative importance either by available data or by intuitive judgment. The relative preferences found by pairwise comparisons are then used to compute weights (priorities) for every part of the hierarchy model. The evaluation computed for all decision alternatives then shows their relative strength from the point of view of the entire problem. It is the advantage of AHP that even considerably diverse criteria can be used in the model, and that not only exact data but also human judgments can be applied to describe various aspects of the problem, [Saaty, 1994].

Formally, AHP is expressed by matrices and matrix operations are used to find and evaluate the best alternative:

Let $A_{1}, A_{2}, . ., A_{n}$ be a set of variables. The quantified judgments on pairs of variables $A_{i}, A_{j}$ are represented by an $n \times n$ matrix $A=\left(a_{i j}\right), i, j=1,2, \ldots, n$. Entries $a_{i j}$ are defined as follows: if $a_{i j}=a$, then $a_{j i}=1 / a ; a \neq 0$ for all $i, j$. As $A_{i}$ is considered to be of equal relative intensity to itself, then $a_{i i}=1$ for all $i$.

$$
A=\left(\begin{array}{cccc}
1 & a_{12} & \cdots & a_{1 n} \\
1 / a_{12} & 1 & \cdots & a_{2 n} \\
\cdots & \cdots & \cdots & \cdots \\
1 / a_{1 n} & 1 / a_{2 n} & \cdots & 1
\end{array}\right)
$$

Then, relative rankings of variables will be obtained by computing an eigenvector $X$ of the matrix $A$.

$$
A X=\lambda X
$$

Finally, by matrix multiplication of matrices of relative rankings of criteria and alternatives relative rankings matrix, total ranking of particular alternatives is obtained.

\section{Tropical algebras}

Two most frequently used tropical algebras are the max-plus and the max-prod algebra. In this paper we work with preference matrices in the multiplicative form. It is natural, therefore, to process the preference matrix in the max-prod algebra. By max-prod algebra we understand a linear structure on a linearly ordered set $\mathcal{R}$ of real numbers together with the binary operations $\oplus=$ maximum and $\otimes=$ product, similarly as the ordinary addition and multiplication operations are used in the classical linear algebra. The operations $\oplus$ and $\otimes$ are extended to matrices and vectors in a natural way. We should 
remark that the max-prod algebra is isomorphic to max-plus algebra, with the operations maximum and addition. The eigenvalue of a given max-plus or max-prod matrix and the eigenvectors can be efficiently described by considering cycles in specifically evaluated directed graphs.

The eigenproblem in the max-prod algebra $(\mathcal{R}, \oplus, \otimes)$ can be formulated as follows. Given matrix $A \in \mathcal{R}^{n \times n}$, find $\lambda \in \mathcal{R}$ and $X \in \mathcal{R}^{n}$ such that

$$
A \otimes X=\lambda \otimes X
$$

It is a well-known fact that the eigenvalue $\lambda$ can be computed as the maximal geometric cycle mean in the complete directed graph $G(A)$ with $n$ nodes, in which the edges are evaluated by the matrix inputs, e.i. the weight of the edge $(i, j)$ is $w(i, j)=a_{i j}$ for every $i, j$. If $C=\left(v_{0}, v_{1}, v_{2}, \ldots, v_{k}\right)$ with $v_{0}=v_{k}$ is a cycle of length $k>0$ in $G(A)$, then the weight of $C$ is the value $w(C)=\prod_{i=1}^{k} w\left(v_{i-1}, w_{i}\right)$, and the geometric cycle mean $\bar{w}(C)=w(C)^{1 / k}$, [Ramík \& Korviny, 2010]. Hence $\lambda=\max _{C \text { cycle in } G(A)} \bar{w}(C)$. The maximal geometric cycle mean can be computed in $O\left(n^{3}\right)$ time be slightly modified Karp's method, see [Karp, 1978].

When the eigenvalue $\lambda$ has been computed, then we denote $B=A \otimes \lambda^{-1}$ and compute the matrix $B^{\star}=I \oplus B \oplus B^{2} \oplus \cdots \oplus B^{n-1}$, which is called Kleene star. The columns of the Kleene star matrix with diagonal value 1 are the fundamental eigenvectors of $B$ with eigenvalue value 1 , and they are also the eigenvectors of the original matrix $A$ with eigenvalue $\lambda$.

\section{Consistent matrix}

In this section we define the consistency of matrices. Consistent matrix is a form of preference matrix where the following conditions are satisfied.

1. $a_{i i}=1$

2. $a_{i j}=1 / a_{j i}$

3. $a_{i j} * a_{j k}=a_{i k}$

It should be remarked that Kleene star matrix $B^{\star}=\left(A \otimes \lambda^{-1}\right)^{\star}$ is consistent. Conversely, if a preference matrix $A$ is consistent, then it is equal to its Kleene star matrix, moreover, all eigenvectors are multiples of each other. Thus, for a consistent matrix there exists only one fundamental eigenvector, which gives the priority vector, after normalization.

Example 1. The eigenproblem for a given matrix $A \in \mathcal{R}^{n \times n}$ in extremal (max-prod) algebra consists of finding a vector $x \in \mathcal{R}^{n}$ (eigenvector) such that the equation $A \otimes x=\lambda \otimes x$ holds true. The solutions are found by LibNOM (Library of Nonstandard Optimization Methods - developed at the University of Hradec Králové, Czech Republic, 2012).

$$
A=\left(\begin{array}{ccc}
1 & 1 / 2 & 3 \\
2 & 1 & 6 \\
1 / 3 & 1 / 6 & 1
\end{array}\right)
$$

$\lambda=1$ and eigenspace of fundamental eigenvector found by Kleene star method $\left(B^{\star}=I \oplus B \oplus B^{2}\right)$ in LibNOM is

$$
\left(\begin{array}{lll}
1 & 0 & 0 \\
0 & 1 & 0 \\
0 & 0 & 1
\end{array}\right) \oplus\left(\begin{array}{ccc}
1 & 0.5 & 3 \\
2 & 1 & 6 \\
0.3333 & 0.1667 & 1
\end{array}\right) \oplus\left(\begin{array}{ccc}
1 & 0.5 & 3 \\
2 & 1 & 6 \\
0.3333 & 0.1667 & 1
\end{array}\right)
$$

Kleene star matrix $B^{\star}$ is consistent with $\lambda=1$ and normalized fundamental eigenvector $x$ is

$$
B^{\star}=\left(\begin{array}{ccc}
1 & 0.5 & 3 \\
2 & 1 & 6 \\
0.3333 & 0.1667 & 1
\end{array}\right) \quad X=\left(\begin{array}{l}
0,3 \\
0,6 \\
0,1
\end{array}\right)
$$




\section{Inconsistent matrix}

For an inconsistent matrix $A$ the priority vector is found in the following steps.

1. Find the eigenvalue $\lambda$ of matrix $A$. The eigenvalue is maximal geometric mean weight of cycle in the corresponding graph, computation is done by the modified Karp's algorithm

2. Converte $A$ into matrix $B=A \otimes \lambda^{-1}$ to get the eigenvalue equal to one.

3. Compute the Kleene star matrix $B^{\star}=I \oplus B \oplus B^{2} \oplus \cdots \oplus B^{n-1}$, a consistent matrix.

4. Normalize the fundamental eigenvector, any column from $B^{\star}$. The normalized column is the priority vector.

Example 2. The example is based on an actual problem: how to choose a conference for presentation of research results. We prepare a 4 attributes and 3 alternatives, as is shown in a picture bellow.

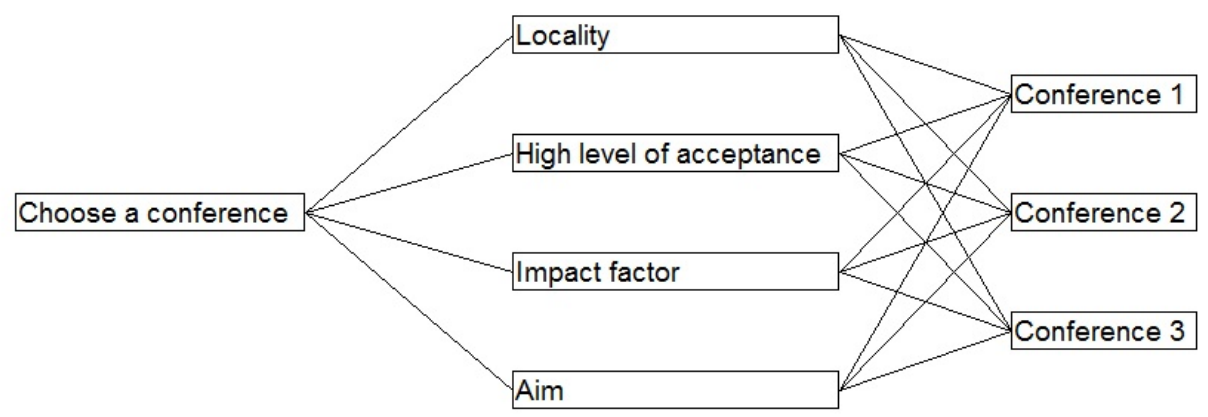

Figure 1: Hierarchy graph

The problem is situated as a way, how to choose a conference. We defined a four levels

- Locality - exotic, holiday areas, etc.,

- High level of acceptance,

- Impact factor - means the outputs of the conference, from nonindexed proceedings to journal with impact factor,

- Aim - if the conference is very close to my work.

We anonymised the conference, but there is a list of short information which will described the instance.

Conference 1 is located on very interesting places, in most cases oceanic places, the level of acceptance is almost $95 \%$, the outputs have almost zero value for current scientific scoring system, the conferences are wide, with many scientific areas.

Conference 2 is located on interesting places, not necessary close to ocean, the level of acceptance is high in case of not so wide target scientific population. The outputs have moderate importance, but the level of papers and discussions on the conference allows you to prepare a journal paper. The aim is very close.

Conference 3 is located at normal places and it is easy to travel on it. The level of acceptance is higher. The outputs of the conference are indexed in required databases and the aim is close. 
In the example are used two programs Criterium Decision Plus and our LibNOM working with the extremal algebra max-prod.

Both approaches use a pairwise comparisons, where the priorities will be derived from a series of measurements: pairwise comparisons involving all the nodes. The nodes at each level will be compared, two by two, with respect to their contribution to the nodes above them. The results of these comparisons will be entered into a matrix which is processed mathematically to derive the priorities for all the nodes on the level.

\begin{tabular}{|c|l|}
\hline Intensity of Importance & Definition \\
\hline 1 & Equal importance \\
3 & Moderate importance \\
5 & Strong importance \\
7 & Very strong importance \\
9 & Extreme importance \\
\hline
\end{tabular}

The following matrices represent the preference matrices with transferred weight.

Locality matrix is inconsistent matrix, has a $\lambda=1.1587$ and normalized fundamental eigenvector is

$$
\text { Locality }=\left(\begin{array}{ccc}
1 & 7 & 9 \\
1 / 7 & 1 & 2 \\
1 / 9 & 1 / 2 & 1
\end{array}\right) \quad X=\left(\begin{array}{c}
0.7928 \\
0.1312 \\
0.0760
\end{array}\right)
$$

High level of acceptability matrix is inconsistent matrix, has a $\lambda=1.3867$ and normalized fundamental eigenvector is

$$
\mathrm{HLA}=\left(\begin{array}{ccc}
1 & 9 & 8 \\
1 / 9 & 1 & 1 / 3 \\
1 / 8 & 3 & 1
\end{array}\right) \quad X=\left(\begin{array}{c}
0.7978 \\
0.0639 \\
0.1383
\end{array}\right)
$$

Impact factor matrix is inconsistent matrix, has a $\lambda=1.3867$ and normalized fundamental eigenvector is

$$
\mathrm{IF}=\left(\begin{array}{ccc}
1 & 1 / 9 & 1 / 8 \\
9 & 1 & 3 \\
8 & 1 / 3 & 1
\end{array}\right) \quad X=\left(\begin{array}{l}
0.0519 \\
0.6484 \\
0.2997
\end{array}\right)
$$

Aim matrix is inconsistent matrix, has a $\lambda=1.1587$ and normalized fundamental eigenvector is

$$
\operatorname{Aim}=\left(\begin{array}{ccc}
1 & 1 / 9 & 1 / 7 \\
9 & 1 & 2 \\
7 & 1 / 2 & 1
\end{array}\right) \quad X=\left(\begin{array}{l}
0.0572 \\
0.5969 \\
0.3458
\end{array}\right)
$$

Criteria matrix is inconsistent matrix, has a $\lambda=1.1006$ and normalized fundamental eigenvector is

$$
\text { Criteria }=\left(\begin{array}{cccc}
1 & 1 / 6 & 1 / 9 & 1 / 3 \\
6 & 1 & 1 / 2 & 2 \\
9 & 2 & 1 & 4 \\
3 & 1 / 2 & 1 / 4 & 1
\end{array}\right) \quad X=\left(\begin{array}{c}
0.0524 \\
0.2857 \\
0.5191 \\
0.1428
\end{array}\right)
$$

The linear algebra approach as an output of the program Criterium Decision Plus is below.

$$
\begin{gathered}
\text { Locality }=\left(\begin{array}{l}
0.793 \\
0.131 \\
0.076
\end{array}\right) \text { HLA }=\left(\begin{array}{l}
0.798 \\
0.064 \\
0.138
\end{array}\right) \text { IF }=\left(\begin{array}{l}
0.052 \\
0.648 \\
0.300
\end{array}\right) \quad \text { Aim }=\left(\begin{array}{l}
0.057 \\
0.597 \\
0.346
\end{array}\right) \\
\text { Criteria }=\left(\begin{array}{l}
0.051 \\
0.282 \\
0.526 \\
0.141
\end{array}\right)
\end{gathered}
$$

By comparing the obtained values we see that both methods give almost identical results in this case. 


\section{Conclusions}

Relative importance of alternatives is computed from the (possibly incosistent) preference matrix in AHP multi-criteria decision problem as the eigenvector of the preference matrix by the methods used in max-prod algebra. Given preference matrix is processed by max-prod operations, until a steady state is reached. The eigenvector of the matrix then respects all preference relations expressed in the preference matrix. Efficient algorithms for computing eigenvectors in the tropical algebra are described.

Further research in this direction will consider possible use of other non-standard algebras, such as tropical or fuzzy algebra. Binary operation maximum and plus, or similar combinations with minimum instead of maximum could be used. Similar approach will involve the fuzzy algebras with the operation maximum together with some of fuzzy triangular norms, e.g. the Gödel, Lukasiewicz, product, or drastic t-norm.

\section{Acknowledgements}

The support of Czech Science Foundation GAČR \#402/09/0405 and Grant Agency of Excellence UHK FIM \#2214 is kindly acknowledged.

\section{References}

[Brualdi \& Ryser, 1991] Brualdi, R. A.,Ryser, H. J. (1991). Combinatorial Matrix Theory, in: Encyclopaedia of Mathematics and its Applications, vol. 39, Cambridge Univ. Press, Cambridge, 1991.

[Butkovič, 2010] Butkovič, P. (2010). Max-linear Systems: Theory and Algorithms, Springer Monographs in Mathematics, Springer-Verlag, 2010.

[Carlsson \& Fullér, 1996] Carlsson, C., Fullr, R. (1996). Fuzzy multiple criteria decision making: Recent developments, Fuzzy Sets and Systems, Volume 78, Issue 2, 11 March 1996, Pages 139-153.

[Cuninghame-Green, 1976] Cuninghame-Green, R. A. (1976). Minimax algebra, Lecture Notes in Econom. and Math. Systems 166, Springer-Verlag, Berlin, 1979.

[Dytczak \& Szklennik, 2011] Dytczak, M., Szklennik, N. (2011). Principles and applications of AHP/ANP - based multiple MCDA methods approach, in: ISAHP 2011. Proceedings, Sorrento, 15-18 June 2011.

[Rashid et al., 2012] Rashid, I., Gavalec, M., Sergeev, S. (2012). Eigenspace of a three-dimensional maxLukasiewicz fuzzy matrix, Kybernetika 48 (2012), 309-328.

[Gavalec et al., submitted] Gavalec, M., Rashid, I., Cimler, R. (submitted). Eigenspace structure of a max-drast fuzzy matrix, Fuzzy Sets and Systems (submitted).

[Heidergott et al., 2006] Heidergott, B., Olsder, G.J., van der Woude, J. (2006). Max Plus at Work, Princeton University Press, Princeton, New Jersey, 2006.

[Karp, 1978] Karp, R.M. (1978). A characterization of the minimum cycle mean in a digraph, Discrete Math. 23 (1978), 309-311.

[Leung \& Cao, 2000] Leung, L.C., Cao, D. (2000). On consistency and ranking of alternatives in fuzzy AHP, European Journal of Operational Research, Volume 124, Issue 1, 1 July 2000, Pages 102-113.

[Mls \& Gavalec, 2009] Mls, K. and Gavalec, M. (2009). Multi-criteria models in autonomous decision making systems. In Proceedings of the 10th International Symposium on the Analytic Hierarchy/Network Process. pp 1-8, University of Pittsburgh, Pittsburgh, Pennsylvania, USA.

[Olsder, 1991] Olsder, G. (1991). Eigenvalues of dynamic max-min systems, in Discrete Events Dynamic Systems 1, Kluwer Academic Publishers, 1991, 177-201.

[Ramík \& Korviny, 2010] Ramík, J., Korviny, P. (2010). Inconsistency of pair-wise comparison matrix with fuzzy elements based on geometric mean, Internat. J. Fuzzy Sets and Systems 161(2010), 16041613. 
[Ramík \& Perzina, 2010] Ramík, J., Perzina, R. (2010). A method for solving fuzzy multicriteria decision problems with dependent criteria, Fuzzy Optimization and Decision Making, Springer Verlag,Vol.9, No.2, 123-141.

[Saaty, 1980] Saaty, T.L. (1980). The analytic hierarchy process. McGraw-Hill, New York.

[Saaty, 1994] Saaty, T.L. (1994). Fundamentals of decision making. Pittsburgh: RWS Publications.

[Saaty, 2003] Saaty, T.L. (2003). Decision-making with the AHP: Why is the principal eigenvector necessary, European Journal of Operational Research, Volume 145, Issue 1, 16 February 2003, Pages 85-91. 